\title{
Asociación entre el consumo de macronutrientes de la dieta y caries en adultos y adultos mayores diabéticos tipo 2
}

\section{Association between dietary macronutrients and caries in type 2 diabetic adults and elderly}

\author{
Giacaman RA ${ }^{1}$, Rojas Quintanilla $\mathrm{T}^{1}$, Soto Poblete $\mathrm{A}^{2}$
}

\begin{abstract}
RESUMEN
Objetivos: Se ha reportado una mayor tasa de caries en pacientes con diabetes mellitus tipo 2 (DM), probablemente debido a cambios en los patrones de dieta. El objetivo de este estudio fue determinar si existe una asociación entre la composición de macronutrientes de la dieta y la caries en sujetos con DM. Métodos: Una muestra compuesta por 33 sujetos con DM y 37 controles sin la patología fue examinada para determinar la experiencia de caries mediante el índice COPD y la prevalencia de caries radiculares con ICDAS II. Se aplicó una encuesta de dieta de reporte de 24 horas. Para determinar la composición de la dieta, los alimentos fueron clasificados acorde al contenido de macronutrientes; carbohidratos, proteínas y lípidos (gramos/día, kilocalorías y porcentaje del nutriente/día). Resultados: Los resultados fueron analizados mediante Kruskal-Wallis y Mann-Whitney. Un análisis de correlación y un modelo de regresión lineal se utilizaron para analizar la asociación entre composición de macronutrientes y la tasa de caries coronales y radiculares, con un nivel de significancia del 95\%. Resultados: Los pacientes diabéticos tipo 2 mostraron mayor número de dientes perdidos por caries, más dientes obturados y más caries radiculares $(p<0.05)$ que los controles sin DM. Los diabéticos presentaron menor consumo de carbohidratos $(p=0.021)$, pero mayor consumo de proteínas $(p=0.0405)$ que los controles. Se verificó una asociación directa entre un mayor consumo de proteínas con una tasa mayor de caries radiculares en pacientes con $D M(p<0.001)$. Conclusión: Las diferencias en el consumo de macronutrientes en pacientes con DM no se relacionan con tasas diferenciales de caries coronales, pero un mayor consumo de proteínas se asocia con una mayor prevalencia de caries radiculares.
\end{abstract}

Rev. Clin. Periodoncia Implantol. Rehabil. Oral Vol. 5(2); 78-82, 2012.

Palabras clave: Caries dental, caries radicular, diabetes, nutrientes, grasas, carbohidratos, proteínas, dieta, índice COPD, ICDAS II.

\begin{abstract}
Objectives: Higher caries rates have been reported for type 2 diabetes mellitus patients (DM). Changes in dietary patterns may be partly responsible. The aim of this study was to examine a potential association between dietary macronutrient composition and caries experience in DM patients. Methods: Thirty three adults with DM and thirty seven controls, not affected by DM, were examined to assess coronal caries through DMFT index and root caries by ICDAS II. A 24-hr diet recall survey was used to determine dietary macronutrient composition supplemented with standardized tables and expressed as grams /day, kilocalories/nutrient and percentage of nutrient/day. Results were compared using Kruskal-Wallis and Mann-Whitney. A correlation analysis was performed and a linear regression model was built for a potential association between macronutrient composition and coronal or root caries. A 95\% significance level was set. Results: DM patients showed lower remaining teeth, more fillings and more root caries ( $p<0.05)$ than controls. Lower carbohydrate consumption $(p=0.021)$, but higher protein intake $(p=0.0405)$ was observed in DM patients as compared with controls. A statistically significant association between an increased protein consumption and higher percentage of root caries was found in DM patients ( $p<0.001$ ). Conclusion: Although differences in macronutrient consumption in DM patients are not correlated with differential rates of coronal caries, higher protein consumption appears to be associated with higher prevalence of root caries.
\end{abstract}

Rev. Clin. Periodoncia Implantol. Rehabil. Oral Vol. 5(2); 78-82, 2012.

Key words: Dental caries, root caries, diabetes, nutrients, dietary fats, dietary carbohydrates, dietary proteins, diet, DMFT index, ICDAS II.

\section{INTRODUCCIÓN}

La diabetes mellitus tipo 2 (DM) es un síndrome hereditario o adquirido que afecta el metabolismo de carbohidratos, grasas y proteínas, lo cual resulta en complicaciones agudas y crónicas debido a la falta relativa o absoluta de insulina ${ }^{(1)}$. Se ha señalado en la literatura disponible que las enfermedades sistémicas como la DM pueden alterar el medio ambiente oral favoreciendo la aparición de enfermedades o empeorando condiciones preexistentes ${ }^{(2)}$. La evidencia reportada muestra que la DM afecta a la salud oral, incrementado el riesgo de patologías orales incluyendo infecciones agudas y posiblemente lesiones malignas y premalignas de la mucosa oral(2). Asimismo, la DM muestra una asociación con mayor riesgo de cursar enfermedades periodontales ${ }^{(3-6)}$ y la inflamación generada por dicha patología oral puede incrementar la resistencia sistémica a la insulina(7).

Existe la idea en el ambiente odontológico que la caries dental ha dejado de ser un problema en los países desarrollados, cuando en realidad afecta entre el $60 \%$ al $90 \%$ de la población escolar y a la gran mayoría de los adultos ${ }^{(8)}$, con una prevalencia muy alta en Latinoamérica. Numerosos factores explican la susceptibilidad individual a la enfermedad, de ahí que se la considerada tradicionalmente una enfermedad multifactorial, incluyendo tanto factores locales como sistémicos ${ }^{(9)}$. A igual que lo que ocurre en la periodontitis, se ha especulado acerca del rol que podrían tener las patologías sistémicas en la caries. En particular, la DM podría jugar un rol, toda vez que genera cambios en el ecosistema oral, los que podrían afectar la susceptibilidad a la caries dental. Pese a lo anterior, la asociación entre DM y un mayor riesgo de caries no ha sido bien establecida, ya que mientras algunos autores señalan una leve mayor tasa de caries entre pacientes diabéticos ${ }^{(10)}$, otros estudios no han encontrado una asociación significativa ${ }^{(3,11,12)}$. Se ha reportado que los pacientes diabéticos tendrían mayor prevalencia de caries radiculares, pero no coronarias ${ }^{(13)}$. Una mayor prevalencia de enfermedad periodontal en pacientes afectados por DM podría condicionar lo anterior. De hecho, nosotros hemos reportado que el aumento de la severidad

1. Cariology Unit. Department of Oral Rehabilitation. University of Talca. Talca, Chile.

2. Instituto de Matemática y Física. Universidad de Talca. Talca, Chile.

Correspondencia autor: Rodrigo A. Giacaman. giacaman@utalca.cl. Escuela de Odontología, Universidad de Talca. 2 Norte 685, Talca, Chile. Trabajo recibido el 03/05/2012. Aprobado para su publicación el 04/07/2012. 
de la enfermedad periodontal se correlaciona con un aumento en el recuento de Streptococcus mutans ${ }^{(14)}$. Diferencias en la composición de la microflora oral podría ser un mecanismo potencial para explicar una supuesta mayor prevalencia de caries radiculares y periodontitis en pacientes diabéticos ${ }^{(15)}$. Por otra parte, y en contraste con lo anterior, una investigación sugiere que pacientes con DM tendrían menos caries que los no diabéticos debido a una dieta restringida en hidratos de carbono, lo que actuaría como un factor protector ${ }^{(16)}$.

La dieta como factor etiológico de la caries ha sido enfocada principalmente al consumo de hidratos de carbono simples, particularmente la sacarosa ${ }^{(17)}$. Pese a ello, desde hace bastante tiempo se ha señalado que los demás nutrientes de la dieta juegan un importante rol en la enfermedad ${ }^{(18)}$, pero éstos no han sido estudiados suficientemente. Dado que la composición de la dieta puede ser relevante para explicar tasas diferenciales de caries entre pacientes diabéticos y no diabéticos, el objetivo de esta investigación fue determinar si existe una asociación entre la composición de la dieta, en términos de sus macronutrientes y la experiencia de caries en pacientes con DM tipo 2.

\section{SUJETOS Y MÉTODOS}

\section{Sujetos}

Se invitó a participar a pacientes mayores de 15 años con diagnóstico de DM de al menos dos años de antigüedad y a personas de su círculo social, pero libres de la enfermedad que servirían de controles. Los pacientes eran atendidos en consultorios $u$ hospitales de Talca y San Javier en la Región del Maule, Chile. De entre los voluntarios que accedieron a participar y que cumplían con todos los criterios de inclusión se seleccionó una muestra por conveniencia de pacientes y controles: treinta y tres sujetos diagnosticados con DM tipo 2, acorde a criterios internacionales ${ }^{(19)}$, cuyo diagnóstico había sido realizado al menos con dos años de anterioridad y treinta y siete pacientes de control pareados por género fueron finalmente incluidos en el estudio. Al momento del examen los individuos con DM debían estar compensados y en tratamiento. Para determinar la compensación, cada paciente debía contar con un examen de hemoglobina glicosilada de no más de 3 a 4 meses de antelación. En caso de no contar con dicho examen, una nueva hemoglobina glicosilada fue solicitada. Los pacientes controles debían haber sido examinados por un médico que verificara la ausencia de DM. No obstante, no fueron excluidos de este grupo aquellos pacientes diagnosticados con hipertensión arterial, obesidad o dislipidemia, ya que los diabéticos usualmente presentan esas comorbilidades. Además de la ausencia de DM, los controles debían provenir de un nivel social similar, lo que se garantizó solicitando a los diabéticos que acudieran acompañados de los controles invitados por ellos mismos desde sus contactos. Con lo anterior se intentó hacer que los controles tuviesen hábitos y nivel educacional similares. Si bien es cierto no se pareó el estudio por edad, se intentó que los controles tuviesen edades similares a los casos. Se excluyeron del estudio, tanto pacientes con DM como sus controles que tuvieran patologías sistémicas graves. Los examinadores no fueron ciegos a la condición diabética de los participantes. El tamaño de la muestra se estimó con un nivel de confianza 95\% con 5\% de error (Raosoft, Inc.). El protocolo del estudio junto con el consentimiento informado, el que debía ser firmado por cada participante, fue aprobado por el Comité de Bioética de la Universidad de Talca.

\section{Determinación de la Prevalencia de Caries}

Se realizó un examen clínico intraoral en el sillón dental apoyado por iluminación auxiliar del equipo dental. Los dos examinadores que registraron los datos fueron previamente entrenados y calibrados hasta obtener una adecuada reproducibilidad mediante un índice kappa de más de 0.75 utilizando COPD. El índice de caries coronales utilizado fue el COPD, obtenido según los criterios de la OMS ${ }^{(20)}$. Para obtener la prevalencia de caries radiculares se emplearon los criterios ICDAS II para este tipo de lesiones ${ }^{(21)}$. Se registraron las lesiones de caries coronales y radiculares por separado y por cada superficie afectada. Para las caries radiculares se registró el código mayor en caso que hubiese más de una superficie comprometida. Los cálculos del porcentaje de caries de cada paciente se hicieron considerando el número de dientes remanentes para evitar sesgos en la interpretación de los números netos de lesiones. Así, se dividió el número de caries por el total de dientes presentes multiplicado por 100 .

\section{Determinación de la Composición de la Dieta}

La dieta fue evaluada en términos de su contenido de macronutrientes. Para obtener los datos de dieta de los participantes se utilizó la encuesta de dieta reporte de 24 horas. Esta es una encuesta guiada sobre los alimentos consumidos durante las 24 horas anteriores al examen ${ }^{(22)}$, incluyendo el tipo de alimento que se consume y la cantidad de ellos. Durante la realización de la encuesta se mostraron a los pacientes distintos contenedores (platos, vasos y tazas) de muestra para ayudar a la cuantificación de las cantidades y las porciones. Para determinar la calidad y la composición de los alimentos consumidos y con ello el aporte calórico consumido se emplearon tablas estandarizadas para la población chilena ${ }^{(23)}$. Cada macronutriente; hidratos de carbono, proteínas y lípidos, fueron cuantificados acorde a las cantidades consumidas en gramos, kilocalorías y en términos del porcentaje que cada uno representaba de la dieta total. Además, se categorizó a los pacientes según si su consumo de macronutrientes estaba dentro, bajo o por sobre las recomendaciones internacionales ${ }^{(24)}$.

\section{Análisis Estadístico}

Se realizaron pruebas de normalidad para evaluar la distribución de los datos. Dada la distribución no normal de los datos, las diferencias en cuanto a la prevalencia de caries y los porcentajes de consumo de macronutrientes entre ambos grupos en estudio fueron comparadas mediante el test no-paramétrico de Kruskal-Wallis, seguido de Mann-Whitney. Las diferencias fueron consideradas significativas si el valor $p$ era menor a 0.05 . Para asociar las variables consumo de macronutrientes y caries se realizó un análisis de correlación de Pearson, para los diferentes macronutrientes como variable independiente y la prevalencia de caries como dependiente. Para las variables que mostraron correlaciones significativas $(p<0.01)$ se construyó un modelo de regresión lineal. Todos los cálculos y análisis estadísticos fueron desarrollados con el programa SPSS 15.0 para Windows.

\section{RESULTADOS}

Los participantes no mostraron diferencias $(p>0.05)$ en cuanto a la edad de hombres $(n=23)$ y mujeres $(n=47)$, con $53.4( \pm 8.8)$ y 54.4 $( \pm 8.4)$ años, respectivamente. Sin embargo, los diabéticos eran mayores que los controles $(p<0.05)$. Mientras los primeros tenían un promedio de $58.1 \pm 8.4$ años de edad, los segundos tenían $50.38 \pm 6.7$ años en promedio (Tabla 1)

Tabla 1. Edad de los participantes, según su condición de DM.

\begin{tabular}{|c|c|c|c|}
\hline \hline Grupo & $\mathbf{n}$ & Edad promedio (DE) & $\begin{array}{c}\text { Valor } \\
\mathbf{p}\end{array}$ \\
\hline Diabéticos & 33 & $58.1(8.4)$ & \multirow{2}{*}{$<0.05$} \\
\hline Controles & 37 & $50.3(6.7)$ & \\
\hline
\end{tabular}

Los pacientes con DM tenían 15.2 dientes en promedio, mientras que los controles no diabéticos tenían $19.8(p<0.05)$ (Figura 1). Al comparar la historia de caries (COPD) de ambos grupos, no se observaron diferencias significativas entre ambos grupos en cuanto al valor del índice (Tabla 2). Pese a ello, al desglosar los componentes de índice (Tabla 2), los pacientes con DM tenían más dientes obturados que los controles $(p<0.05)$, pero los demás componentes del índice no mostraron diferencias estadísticamente significativas entre ambos grupos. En relación al porcentaje de caries radiculares, se calculó mediante el porcentaje del número de caries diagnosticadas dividido por el total de dientes presentes. Los pacientes aquejados de DM tenían significativamente mayor porcentaje de caries radiculares, con un promedio de $14.64 \%$, que los controles, los que sólo tenían un $3.09 \%$ $(p<0.05)$ (Figura 2A). Cuando las caries radiculares fueron clasificadas mediante ICDAS II, no se detectaron diferencias en cuanto a las superficies expuestas sin caries radicular entre los grupos (Figura 2B) Los pacientes con DM, sin embargo, mostraron más caries radiculares, tanto de códigos 1 como 2 , que los controles $(p<0.05)$ (Figura 2B). 
Tabla 2. Índice COPD para caries coronales y sus componentes en los participantes, según condición diabética. Promedio (DE).

\begin{tabular}{|c|c|c|c|}
\hline & Diabéticos & Controles & Valor $\mathbf{p}$ \\
\hline COPD & $14.3(6.7)$ & $14.7(5.3)$ & 0.3835 \\
\hline C & $2.9(2.9)$ & $2.8(2.6)$ & 0.4305 \\
\hline O & $8.8(7.0)$ & $7.0(5.5)$ & $0.0215^{\star}$ \\
\hline P & $3.2(3.5)$ & $5.2(4.5)$ & 0.1190 \\
\hline
\end{tabular}

${ }^{*}=$ valor $\mathbf{p}<0.05$.

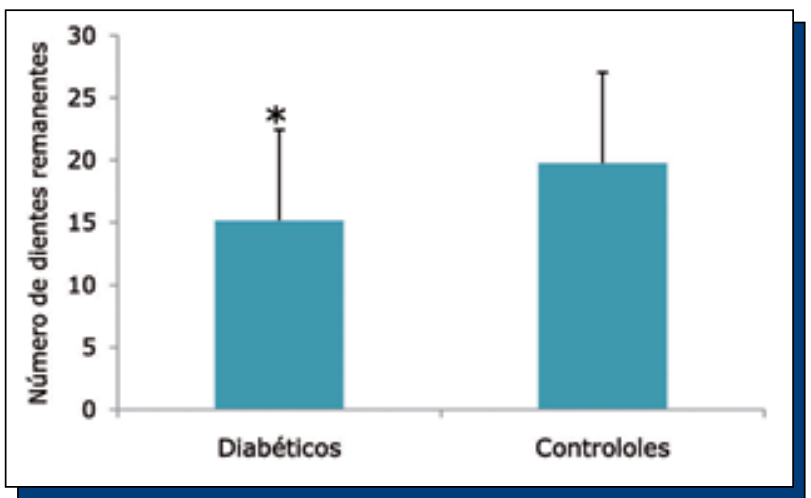

Figura 1. Promedio de dientes en diabéticos y no diabéticos. Las barras indican el promedio de dientes para cada grupo. Las barras de error indican la desviación estándar. ${ }^{*}=p<0.05$.

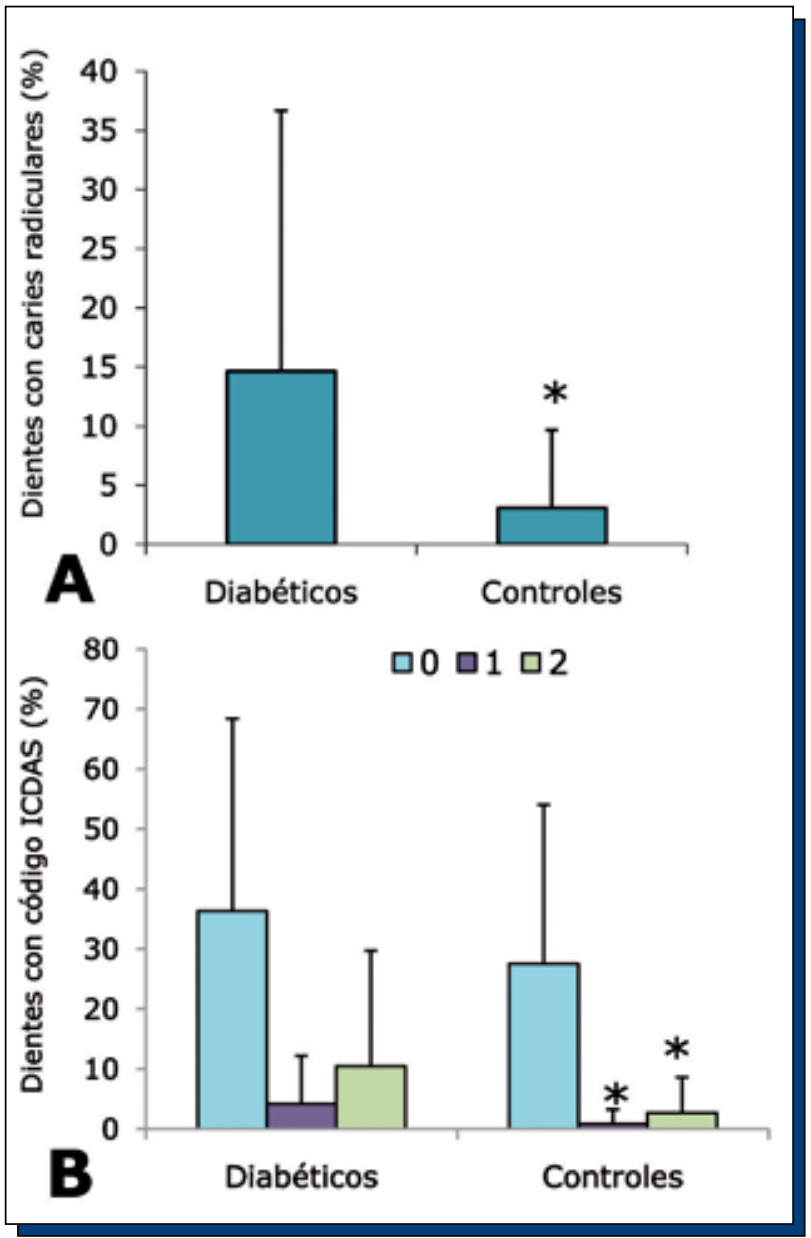

Figura 2. Caries radiculares en pacientes diabéticos y no diabéticos. La figura muestra el porcentaje de caries radiculares, independientemente de su severidad en el panel (A) y las superficies expuestas sin caries (0), con caries de menos de $0.5 \mathrm{~mm}$ (1) y de más de $0.5 \mathrm{~mm}$ (2) en el panel (B). Las barras indican el promedio del porcentaje de caries radiculares del total de dientes presentes para cada grupo. Las barras de error indican la desviación estándar del promedio. * $=p<0.05$
Al analizar el consumo de los diferentes macronutrientes entre ambos grupos (Tabla 3), los pacientes con DM tenían un mayor porcentaje de consumo de proteínas que los controles $(p<0.05)$. En relación a los carbohidratos, los diabéticos tuvieron mayor consumo de este macronutriente que los controles $(p<0.05)$. Finalmente, no se detectaron diferencias significativas en cuanto al consumo de lípidos entre ambos grupos ( $p>0.05$ ). Estos resultados fueron similares cuando el análisis fue realizado como porcentaje del macronutriente en la dieta, como gramos consumidos o como kilocalorías del nutriente. Para sintetizar, sólo se muestran los resultados expresados en porcentajes (Tabla 3).

Tabla 3. Consumo de macronutrientes según estado diabético. Porcentaje promedio por macronutriente (DE).

\begin{tabular}{|c|c|c|c|}
\hline \hline & Diabéticos & Controles & Valor $\mathbf{p}$ \\
\hline Proteínas & $17.6(5.2)$ & $15.7(3.7)$ & $0.0405^{*}$ \\
\hline Carbohidratos & $56.3(8.4)$ & $60.8(9.7)$ & $0.0210^{*}$ \\
\hline Lípidos & $27.8(8.2)$ & $24.1(10.2)$ & 0.0530 \\
\hline * $=$ valor $\mathbf{p}<0.05$
\end{tabular}

$\mathrm{Al}$ analizar el porcentaje de los diferentes macronutrientes que componen la dieta y relacionarlos con el porcentaje de caries coronales, no se observan asociaciones significativas $(p<0.05)$ mediante un modelo de correlación lineal. Tampoco se encontraron asociaciones entre los componentes individuales del índice COPD (datos no mostrados).

Dada la mayor prevalencia de caries radiculares en el grupo de los diabéticos (Figura 2A y B), se exploró la hipótesis de que existiese una asociación entre la caries radicular en los pacientes con DM y el consumo de algunos de los macronutrientes de la dieta. Respecto de los controles, los sujetos con DM tenían un consumo diferente de proteínas y carbohidratos, pero no de lípidos (Tabla 3), por lo que se realizó una correlación lineal entre caries radiculares y porcentaje de proteínas y carbohidratos a partir de la dieta total, obteniendo una correlación estadísticamente significativa para el porcentaje de proteínas $(p<0.01)$, pero no para carbohidratos (Tabla 4). Como resultado de la correlación entre caries radiculares y el mayor consumo de proteínas, se llevó a realizó un modelo de regresión lineal que mostró una asociación estadísticamente significativa $(p<0.001)$ entre las variables (Tabla 5$)$. Lo anterior significa que a mayor porcentaje de consumo de proteínas en la dieta de los pacientes diabéticos, mayor cantidad de caries radiculares.

Tabla 4. Correlaciones entre caries radiculares ( $\%$ de caries del total de dientes) y el consumo porcentual de cada macronutriente (\%).

\begin{tabular}{|c|c|c|c|c|c|}
\hline & & $\begin{array}{c}\text { Caries } \\
\text { radiculares }\end{array}$ & Proteínas & Carbohidratos & Lípidos \\
\hline \multirow{2}{*}{$\begin{array}{c}\text { Caries } \\
\text { radiculares }\end{array}$} & $\begin{array}{l}\text { Correlación } \\
\text { de Pearson }\end{array}$ & 1 & $0.313\left({ }^{* *}\right)$ & -0.096 & -0.050 \\
\hline & $\begin{array}{c}\text { Sig. } \\
\text { (bilateral) }\end{array}$ & & 0.008 & 0.430 & 0.680 \\
\hline \multirow{2}{*}{ Proteínas } & $\begin{array}{l}\text { Correlación } \\
\text { de Pearson }\end{array}$ & $0.313\left(^{* *}\right)$ & 1 & -0.152 & $-0.246\left(^{*}\right)$ \\
\hline & $\begin{array}{c}\text { Sig. } \\
\text { (bilateral) }\end{array}$ & 0.008 & & 0.209 & 0.040 \\
\hline \multirow{2}{*}{ Carbohidratos } & $\begin{array}{l}\text { Correlación } \\
\text { de Pearson }\end{array}$ & -0.096 & -0.152 & 1 & $-0.879\left({ }^{\star \star}\right)$ \\
\hline & $\begin{array}{c}\text { Sig. } \\
\text { (bilateral) }\end{array}$ & .430 & .209 & & 0.000 \\
\hline \multirow{2}{*}{ Lípidos } & $\begin{array}{l}\text { Correlación } \\
\text { de Pearson }\end{array}$ & -0.050 & $-0.246\left(^{*}\right)$ & $-0.879\left(^{\star \star}\right)$ & 1 \\
\hline & $\begin{array}{c}\text { Sig. } \\
\text { (bilateral) }\end{array}$ & 0.680 & 0.040 & 0.000 & \\
\hline
\end{tabular}

** La correlación es significativa al nivel 0.01 (bilateral).

* La correlación es significante al nivel 0.05 (bilateral). 
Tabla 5. Modelo de regresión lineal mediante ANOVA para la correlación entre caries radiculares (\% de caries del total de dientes) y el consumo porcentual de proteínas.

\begin{tabular}{|c|c|c|c|c|c|c|}
\hline \multirow{2}{*}{ Modelo } & & $\begin{array}{c}\text { Suma de } \\
\text { cuadrados }\end{array}$ & gl & $\begin{array}{c}\text { Media } \\
\text { cuadrática }\end{array}$ & F & Sig. \\
\hline \multirow{2}{*}{} & Regresión & 3501.814 & 2 & 1750.907 & 7.375 & $0.001(\mathrm{a})$ \\
\cline { 2 - 7 } & Residual & 15907.067 & 67 & 237.419 & & \\
\cline { 2 - 7 } & Total & 19408.881 & 69 & & & \\
\hline
\end{tabular}

a. Variables predictivas: (Constante), Estado diabético, porcentaje de consumo de proteínas.

b. Variable dependiente: Porcentaje de caries radicular del total de dientes.

\section{DISCUSIÓN}

La relación existente entre la DM y la caries dental continúa siendo materia de controversias. Los pacientes con DM examinados en este estudio tenían menos dientes que los controles sin DM (Figura 1). Sin embargo, al análisis del COPD, estos pacientes tienen incluso menos dientes perdidos por caries (Tabla 2). Esta aparente contradicción se explica por el hecho que la mayoría de los dientes perdidos en los diabéticos obedece a razones periodontales y no por caries, tal como se ha reportado previamente ${ }^{(13)}$. Cabe destacar, eso sí, que la edad promedio de los sujetos con DM era mayor que la de los controles. Pese a lo inapropiado de contar con grupos de edades levemente disímiles y al posible sesgo introducido por el factor etario, se decidió utilizar a este grupo control debido a que se priorizó el que provinieran de un entorno social y cultural similar a los pacientes con DM. Los resultados de esta investigación muestran, además, que las personas afectadas por DM tienen una mayor prevalencia de caries radiculares (Figura 2), pero no coronales, que los sujetos no diabéticos, lo que es consistente con otras investigaciones previas ${ }^{(10,13)}$. Pese a no encontrarse diferencias en la experiencia global de caries entre ambos grupos, los pacientes con DM tenían más dientes obturados que los controles. Esto puede deberse a que los pacientes en control tienen mayor acceso a servicios de salud que sus pares no diabéticos. El hecho de encontrarse en control periódico y ser parte de programas para enfermos crónicos permite que los pacientes con DM puedan ser referidos a atención odontológica por otros miembros del equipo de salud. Se debe resaltar que en Chile, los adultos mayores, los que constituían una parte importante de la muestra, tienen acceso a atención odontológica de mayor complejidad y con garantías explícitas sólo cuando tienen la edad de 60 años, a pesar que igualmente pueden recibir atención a otras edades.

La controversia acerca de si los sujetos afectados por DM son más pronos a la caries reside en el hecho que así como en la presente investigación se encontró una asociación entre caries radicular y DM, otros reportes no la han detectado ${ }^{(3,11,12)}$. La caries radicular se produce en la gran mayoría de los casos una vez que las raíces son expuestas al medio oral ${ }^{(25)}$, por ello, no es de extrañar que exista una mayor prevalencia de caries radiculares entre los diabéticos, toda vez que esta población presenta mayores tasas de enfermedad periodontal que sujetos libres de la patología(4).

Una de las teorías más plausibles que explican la mayor prevalencia de caries radiculares en diabéticos es el cambio en la dieta que tienen los pacientes diabéticos, como parte de la consejería que reciben para el control sistémico de su patología de base ${ }^{(16)}$. De hecho, la Asociación Europea para el Estudio de Diabetes y la Asociación Americana de Diabetes proponen una elección adecuada de los alimentos a consumir, con una dieta baja en grasas e hidratos de carbono refinados, además de promover el consumo de grasas monoinsaturadas ${ }^{(26)}$. Si el paciente siguiera total o parcialmente estas indicaciones, se esperaría encontrar un ecosistema oral con condiciones alteradas respecto de controles que no realizan modificaciones alimentarias. En la misma dirección, si se adscribe la Teoría Ecológica de Placa(27), dichos cambios nutricionales pueden tener repercusiones en el biofilm oral y en la consecuente resultante de caries, lo que en este caso, sólo se observa en las caries radiculares. De allí entonces que se buscara explorar la composición de la dieta en diabéticos y asociarla con las tasas de caries coronales y radiculares. Al análisis de la dieta consumida por ambos grupos se observan diferencias significativas en cuanto al consumo de proteínas y carbohidratos, pero no de lípidos (Tabla 3). Los resultados mostraron una asociación entre un aumento en la cantidad de proteínas en la dieta de los diabéticos y mayor número de caries radiculares (Tablas 4 y 5 ). Se debe señalar, que la asociación en la regresión lineal fue débil, lo que impidió la construcción de un modelo más complejo que controlara otras variables que pudiesen sesgar el estudio. Aún así, no existen muchos estudios que hayan asociado el tipo de dieta, en término de macronutrientes, con caries en diabéticos, por lo que estos resultados obligan a profundizar los hallazgos y no permiten realizar comparaciones con otros estudios. Sin bien es cierto que el rol de los carbohidratos en la caries, pero especialmente la sacarosa, ha sido ampliamente investigados ${ }^{(17)}$, el rol de las proteínas de la dieta es mucho más difuso y requiere investigación a la luz de los presentes resultados. Nosotros hemos demostrado un rol protector contra la desmineralización inducida por Streptococcus mutans por parte de algunos ácidos grasos $^{(28)}$. Las proteínas aportadas en forma exógena en la dieta pueden ser degradadas por flora microbiana proteolítica específica presente en el biofilm oral ${ }^{(29)}$. Nuestro grupo abordará el cómo la presencia de proteínas de la dieta puede condicionar un mayor riesgo de caries radiculares en pacientes diabéticos con una serie de experimentos relevantes para intentar esclarecer este tema.

El presente trabajo posee una serie de situaciones derivadas de la naturaleza de este tipo de estudios que requieren de mayor discusión. Por una parte el utilizar una muestra de pacientes diabéticos que cumplan con los criterios de inclusión resulta una tarea laboriosa y demandante. Para evitar una excesiva variabilidad en los sujetos, este estudio decidió sólo incluir a pacientes compensados. Aún así, la muestra fue compuesta por 33 sujetos con DM y 37 controles de similares características, lo que no difiere de otros estudios publicados sobre el tema(3). Nuevos estudios deberían incluir distintos grados de compensación, para así tener una idea del efecto del control glicémico sobre la caries. Por otra parte, los estudios de dieta son complejos en esencia. La alimentación varía grandemente de persona a persona y por ende incrementa la variabilidad de los resultados en este tipo de investigación. El registro, entonces, se torna en un impedimento mayor para la obtención de datos fiables para el análisis ${ }^{(30)}$. Previamente, nuestro grupo demostró que el uso de un diario dietético semanal y un algoritmo para establecer el potencial cariogénico de la dieta no reflejan la historia de caries de una población adulta ${ }^{(31)}$. Esta investigación, por lo tanto, utilizó una estrategia de recolección de datos diferente mediante encuestas recordatorio de 24 horas y el análisis de la composición de macronutrientes. Esta metodología de registro ha sido ampliamente utilizada en nutrición y es considerada hoy en día como la más apropiada para evitar sesgos y recoger los datos de dieta con mayor precisión, a pesar de sus conocidas limitaciones como son la dificultad para evaluar el tamaño de las porciones y las limitaciones derivadas del recordar lo ingerido el día anterior ${ }^{(32)}$

En conclusión, los pacientes diabéticos poseen mayor prevalencia de caries radiculares y tienen aportes diferenciales de macronutrientes, particularmente carbohidratos y proteínas. Los cambios en la dieta evidenciados en los diabéticos pueden ser un factor de riesgo para caries radicular, particularmente el aumento en el consumo de proteínas. Se requiere más investigación para establecer conclusiones más definitivas.

\section{RECONOCIMIENTOS}

Este trabajo formó parte de los requerimientos para la obtención del título de Cirujano-Dentista de TRQ. Los autores agradecen la colaboración del Dr. Víctor Cubillos en la recolección de datos para este estudio. Los autores han contribuido al estudio y han revisado los datos acá presentados. Parte de estos resultados fueron presentados en la IV Reunión Región Latinoamericana y XXIV Reunión Anual División Chilena de la IADR, el 3 y 4 de octubre de 2011 en Santiago, Chile.

\section{CONFLICTOS DE INTERESES}

Los autores no declaran conflictos de interés. Este estudio fue financiado por el proyecto Fondecyt 11100005 a RAG y por el proyecto \#I001068 de la DI, Universidad de Talca. 


\section{REFERENCIAS BIBLIOGRÁFICAS}

1. Ismail-Beigi F. Clinical practice. Glycemic management of type 2 diabetes mellitus. N Engl J Med, 2012; 366(14): 1319-1327.

2. Roglic G, Unwin N, Bennett P, Mathers C, Tuomilehto J, Nag S et al. The burden of mortality attributable to diabetes: Realistic estimates for the year 2000. Diabetes Care, 2005; 28(9): 2130-2135.

3. Collin H, Uusitupa M, Niskanen L, Koivisto A, Markkanen H, Meurman J. Caries in patients with non-insulin-dependent diabetes mellitus. Oral Surg Oral Med Oral Pathol Oral Radiol Endod, 1998; 85(6): 680-685.

4. Campus G, Salem A, Uzzau S, Baldoni E, Tonolo G. Diabetes and periodontal disease: A case-control study. J Periodontol, 2005; 76(3): 418-425.

5. Torrungruang K, Tamsailom S, Rojanasomsith K, Sutdhibhisal S, Nisapakultorn $\mathrm{K}$, Vanichjakvong $\mathrm{O}$ et al. Risk indicators of periodontal disease in older Thai adults. J Periodontol, 2005; 76(4): 558-565.

6. Tsai C, Hayes C, Taylor G. Glycemic control of type 2 diabetes and severe periodontal disease in the US adult population. Community Dent Oral Epidemiol, 2002; 30(3): 182-192.

7. Skamagas M, Breen TL, LeRoith D. Update on diabetes mellitus: Prevention, treatment, and association with oral diseases. Oral Dis, 2008; 14(2): 105-114.

8. Petersen P, Bourgeois D, Ogawa H, Estupinan-Day S, Ndiaye C. The global burden of oral diseases and risks to oral health. Bull World Health Organ, 2005; 83(9): 661-669.

9. González-Cabezas $C$. The chemistry of caries: remineralization and demineralization events with direct clinical relevance. Dent Clin North Am, 2010; 54(3): 469-478.

10. Lin B, Taylor G, Allen D, Ship J. Dental caries in older adults with diabetes mellitus. Spec Care Dentist, 1999; 19(1): 8-14.

11. Bacić M, Ciglar I, Granić M, Plancak D, Sutalo J. Dental status in a group of adult diabetic patients. Community Dent Oral Epidemiol, 1989; 17(6): 313-316.

12. Zielinski M, Fedele D, Forman L, Pomerantz S. Oral health in the elderly with non-insulin-dependent diabetes mellitus. Spec Care Dentist, 2002; 22(3): 94-98.

13. Hintao J, Teanpaisan R, Chongsuvivatwong V, Dahlen G, Rattarasarn C. Root surface and coronal caries in adults with type 2 diabetes mellitus. Community Dent Oral Epidemiol, 2007; 35(4): 302-309.

14. Contardo S, Díaz N, Lobos O, Padilla C, Giacaman RA. Oral colonization by Streptococcus mutans and its association with the severity of periodontal disease in adults. Rev Clin Periodoncia Implantol Rehabil Oral, 2011; 4(1): 9-12.

15. Hintao J, Teanpaisan R, Chongsuvivatwong V, Ratarasan C, Dahlen G. The microbiological profiles of saliva, supragingival and subgingival plaque and dental caries in adults with and without type 2 diabetes mellitus. Oral Microbiol Immunol, 2007; 22(3): 175-181.

16. Tavares M, Depaola P, Soparkar P, Joshipura K. The prevalence of root caries in a diabetic population. $J$ Dent Res, 1991; 70(6): 979-983.
17. Zero DT. Sugars-the arch criminal? Caries Res, 2004; 38(3): 277-285.

18. Kabara JJ, Swieczkowski DM, Conley AJ, Truant JP. Fatty acids and derivatives as antimicrobial agents. Antimicrob Agents Chemother, 1972; 2(1): 23-28.

19. WHO. Definition and diagnosis of diabetes mellitus and intermediate hyperglycemia: Report of a WHO/IDF consultation. Geneva, Switzerland. 2006

20. OMS. Encuestas de salud bucodental. Métodos básicos. Cuarta ed. Ginebra: Organización Mundial de la Salud. 1997.

21. Ismail AI, Sohn W, Tellez M, Amaya A, Sen A, Hasson H et al. The International Caries Detection and Assessment System (ICDAS): An integrated system for measuring dental caries. Community Dent Oral Epidemiol, 2007; 35(3): 170-178.

22. Urteaga Ribbeck C, Pinheiro Fernandes AC, Atalah Samur E. Comparison of results from two dietary interview methods. Arch Latinoam Nutr, 2003; 53(2): 172-127. 23. Jury G, Urteaga C, Taibo M. Composición química de los alimentos de la piramide alimentaria chilena. In: Cqdl alimentos.xls editor: Instituto de Nutrición y Tecnología de los Alimentos, INTA. 2001.

24. Nishida C, Uauy R, Kumanyika S, Shetty P. The joint WHO/FAO expert consultation on diet, nutrition and the prevention of chronic diseases: Process, product and policy implications. Public Health Nutr, 2004; 7(1A): 245-250.

25. Griffin SO, Griffin PM, Swann JL, Zlobin N. Estimating rates of new root caries in older adults. J Dent Res, 2004; 83(8): 634-638.

26. Westman EC, Feinman RD, Mavropoulos JC, Vernon MC, Volek JS, Wortman JA et al. Low-carbohydrate nutrition and metabolism. Am J Clin Nutr, 2007; 86(2): 276-284. 27. Marsh PD. Microbiology of dental plaque biofilms and their role in oral health and caries. Dent Clin North Am, 2010; 54(3): 441-454.

28. Giacaman RA, Jobet P, Castro RJ, Correa G. Poly and monounsaturated fatty acids reduce sucrose-induced enamel demineralization in an artificial caries model. In preparation presented at the Latin-American Region Meeting of the IADR October 3 and 4, 2011 Santiago, Chile. 2012.

29. Hashimoto K, Sato T, Shimauchi H, Takahashi N. Profiling of dental plaque microflora on root caries lesions and the protein-denaturing activity of these bacteria. Am J Dent, 2011; 24(5): 295-299.

30. Moynihan P, Petersen PE. Diet, nutrition and the prevention of dental diseases. Public Health Nutr, 2004; 7(1A): 201-226.

31. Giacaman RA, Fernández CE, Díaz N. Sugar consumption from the diet measured by a cariogenicity scoring system and caries experience in adults. Submitted, 2012.

32. Carroll RJ, Midthune D, Subar AF, Shumakovich M, Freedman LS, Thompson FE et al. Taking advantage of the strengths of 2 different dietary assessment instruments to improve intake estimates for nutritional epidemiology. $A m \mathrm{~J}$ Epidemiol, 2012; 175(4): 340-347. 